\title{
Procedimiento para la obtención de cargas últimas de arcos de perfiles conformados en frío
}

\section{A procedure for determining the load carrying capacity in cold-formed steel arches}

\author{
$\underline{\text { H. Cifuentes-Bulté }}^{(*)}$, F. Medina-Encina ${ }^{(*)}$
}

RESUMEN

En este trabajo se presenta una metodología de cálculo para la determinación de la carga última de arcos circulares con perfiles trapezoidales conformados en frío. Estos elementos de cubierta, sometidos fundamentalmente a esfuerzos de compresión y de directriz curva, deben ser dimensionados teniendo en cuenta la inestabilidad por pandeo. Actualmente, ninguna normativa describe un procedimiento de cálculo para la consideración del pandeo en elementos curvos. La metodología aquí planteada se basa en la filosofía de las curvas europeas de pandeo establecidas en los Eurocódigos. Así, se ha obtenido un coeficiente reductor empírico que considera los efectos del pandeo conjuntamente con imperfecciones geométricas y mecánicas en función de la esbeltez del arco considerado. Para ello, es necesario determinar la carga última experimental de al menos tres arcos, cuyos radios y luces se encuentren lo suficientemente diferenciados.

Palabras clave: Perfiles conformados en frío; arcos circulares; pandeo de arcos; metodología de cálculo; experimental.

\section{ABSTRACT}

This paper deals with a new methodology for the estimation of the strength of circular arches with cold-formed trapezoidal section. These cover elements, mainly subjected to compression stress and with circular curvature should be dimensioned taking into account the possibility of buckling instability. Currently, no standard describes a method for calculating the buckling consideration of curved elements. The methodology proposed herein shares the same philosophy of the European buckling curves method established in the Eurocodes. Thus, an experimental coefficient considering the effects of buckling and geometrical and mechanical imperfections depending on the slenderness of the considered arch have been obtained. For this, it was necessary to determine the experimental strength of at least three arcs, which radius and spans are sufficiently differentiated.

Keywords: Cold-formed steel profiles; circular arches; buckling of arches; calculation procedure; experimental analysis.

(*) Escuela Técnica Superior de Ingeniería - Universidad de Sevilla, España.

Persona de contacto/Corresponding author: bulte@us.es (H. Cifuentes-Bulté)

Cómo citar este artículo/Citation: Cifuentes-Bulté, H., Medina-Encina, F. (2015). Procedimiento para la obtención de cargas últimas de arcos de perfiles conformados en frío. Informes de la Construcción, 67(539): eo94, doi: http://dx.doi.org/10.3989/ic.13.117.

Licencia / License: Salvo indicación contraria, todos los contenidos de la edición electrónica de Informes de la Construcción se distribuyen bajo una licencia de uso y distribución Creative Commons Reconocimiento no Comercial 3.o. España (cc-by-nc). 


\section{INTRODUCCIÓN}

La utilización de chapas nervadas curvadas con perfiles trapezoidales de pequeño espesor es una técnica comúnmente empleada en los cerramientos de cubierta de ciertas edificaciones. La sección transversal de estos productos es obtenida mediante conformado en frío de láminas de acero de pequeño espesor (0,5-1,5 mm) (1). Como se trata de arcos circulares, los esfuerzos a los que se encuentran sometidos serán predominantemente de compresión, acompañado de esfuerzos de flexión de valor más reducido, y que conlleva la necesidad de comprobación frente al fenómeno de inestabilidad por pandeo global del elemento. Por otro lado, el débil espesor de estos elementos, conjuntamente con las tensiones residuales procedentes del proceso de conformación en frío genera la necesidad de un estudio detallado de estos elementos frente a fenómenos de inestabilidad local de abolladura (1). Atendiendo a la clasificación de secciones establecida en los Eurocódigos (2), este tipo de perfiles es predominantemente de clase 4 , lo que pone de manifiesto la existencia de problemas locales de abolladura.

Por tanto, el correcto dimensionamiento de este tipo de elementos estructurales conlleva la consideración en el cálculo de los fenómenos de inestabilidad por pandeo global del elemento y por pandeo local (abolladura). La inmensa mayoría de códigos de aplicación al cálculo de estructuras metálicas consideran el método de las curvas europeas de pandeo para el estudio de la inestabilidad global. En este método, las imperfecciones que producen una reducción considerable de la carga de pandeo real respecto a la teórica, se consideran a través de unos coeficientes de imperfección correspondientes a cada curva de pandeo. En la gran mayoría de normativas se consideran únicamente elementos de directriz recta, existiendo grandes lagunas en casos de elementos de directriz curva como los arcos circulares (3).

El estudio del pandeo de arcos ya ha sido abordado por diversos investigadores. Así, Fung y Kaplan en 1952 (4), Timoshenko y Gere en 1961 (5) y Gjelsvik y Bodner en 1962 (6) ya aportaron soluciones aproximadas para las cargas críticas de pandeo de diferentes tipos de arcos. Sin embargo, aún hoy en día se encuentran publicaciones recientes sobre este tema (7) (8) (9), lo que pone de manifiesto el interés que todavía suscita. No existen unas expresiones que permitan la obtención de la carga crítica de pandeo de un arco con cualquier distribución de carga y condiciones de contorno. Los casos de carga disponibles en la bibliografía y cuyas expresiones pueden resultar de fácil determinación se refieren únicamente a casos de carga puntual (10) y carga en dirección radial uniformemente distribuida sobre el $\operatorname{arco}(11)$.

En este trabajo se presenta un procedimiento de cálculo de la carga última de chapas nervadas con generatriz de arco circular, considerando fenómenos de inestabilidad global por pandeo e inestabilidad local por abolladura. Este procedimiento está basado en resultados experimentales y numéricos, obteniendo la carga última de la chapa curvada en función de su esbeltez y considerando un coeficiente de reducción por pandeo sobre la carga última de la sección eficaz del perfil. Los ensayos experimentales han puesto de manifiesto el fallo por pandeo de este tipo de elementos, permitiendo establecer una función de ajuste experimental para la determinación de la carga última en función de la esbeltez del elemento. Por otro lado, los resultados numéricos han permitido la obtención y constatación de las cargas numéricas de fallo por pandeo global. Mediante el procedimiento aquí descrito se pueden obtener las cargas de utilización de este tipo de cubierta, con una metodología similar a la propuesta en los Eurocódigos (2) para elementos de directriz recta sometidos predominantemente a compresión.

\section{ANTECEDENTES TEÓRICOS}

\subsection{Pandeo de arcos de circunferencia}

Análogamente a la teoría de Euler para el estudio de pandeo en elementos rectos, Bradford et al. (12) y Pi et al. (10) (11) (13) (14) establecieron que el parámetro fundamental para el pandeo de arcos circulares es su esbeltez, definida por:

$$
\lambda=\frac{S_{k}^{2}}{4 \cdot i \cdot R}
$$

Donde $S_{k}$ es la longitud del arco de circunferencia, $i$ es el radio de giro de la sección transversal y $R$ el radio de curvatura del arco. Como se observa en la ecuación [1] el concepto de esbeltez es ligeramente distinto que en el caso de elementos rectos. En arcos circulares se puede dar el caso de elementos con la misma sección transversal y diferentes longitudes y radios que presenten idéntico valor de la esbeltez.

Se considera que un arco de circunferencia puede presentar dos modos de pandeo distintos correspondientes a pandeo simétrico y pandeo asimétrico (Figura 1). El que se produzca antes uno u otro modo de pandeo depende de la esbeltez del elemento (10). Arcos de esbeltez reducida presentarán modo de pandeo simétrico frente a arcos de esbeltez elevada donde la carga crítica menor es la correspondiente a pandeo asimétrico (12). Así, en arcos circulares de pequeña esbeltez, con una gran rigidez a flexión el modo de pandeo preferente será simétrico, siendo el valor del axil crítico:

$$
N_{c r \cdot s}=\frac{\pi^{2} E I_{x}}{S^{2}}
$$
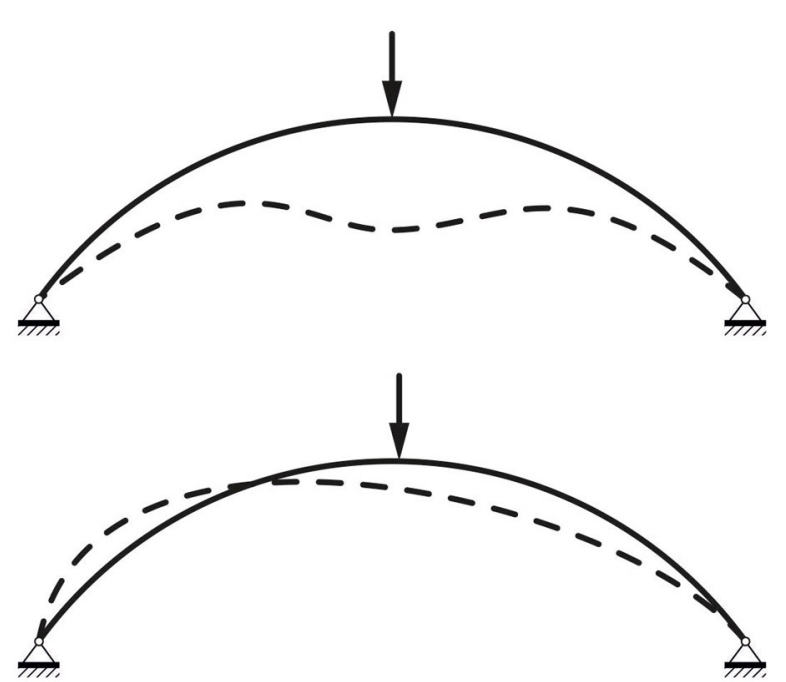

Figura 1. Pandeo simétrico de un arco circular (sup.) y pandeo asimétrico (inf.) 
En aquellos casos donde la esbeltez del arco sea elevada, el primer modo de pandeo corresponderá a un pandeo asimétrico, siendo el valor del axil crítico en este caso:

$$
N_{c r \cdot a}=\frac{\pi^{2} E I_{x}}{(S / 2)^{2}}
$$

Esto pone de manifiesto que el modo y la carga crítica de pandeo del elemento dependen de su esbeltez, así como de la forma de aplicación de la carga exterior que introduce el esfuerzo de compresión en el arco. Las expresiones [2] y [3] son válidas para arcos de directriz circular biarticulados sometidos a una presión radial uniforme.

Como se desprende de las ecuaciones [2] y [3], el valor de la carga crítica menor es la correspondiente a pandeo simétrico. Por tanto, el que en arcos esbeltos el primer modo de pandeo se deba a una forma asimétrica tiene su explicación mediante un cálculo de segundo orden, considerando no linealidades geométricas. Los estudios de Pi et al. (10) y de Bradford et al. (12) demostraron una gran influencia de la forma de la sección. Así, en secciones esbeltas como la analizada en este estudio, el modo de pandeo será predominantemente asimétrico, excepto en casos de valor reducido de la longitud entre apoyos del arco (15).

Por otro lado, como es ya sabido, el valor de las cargas críticas reales es bastante inferior al valor crítico teórico (lineal o no lineal). Esta diferencia que es debida a imperfecciones geométricas y mecánicas es tanto mayor cuanto mayor es la esbeltez del elemento (16). Este fenómeno es recogido por la metodología del Eurocódigo 3 para elementos rectos, no encontrándose referencia alguna para el caso de elementos con directriz curva. En este sentido, $\mathrm{Ku}-$ ranishi y Yakubi (17) y Verstappen et al. (18) propusieron un criterio de diseño considerando elementos rectos en la comprobación a pandeo de arcos que no produce resultados satisfactorios (19).

\subsection{Abolladura en perfiles conformados en frío}

Los perfiles conformados en frío se caracterizan principalmente por su pequeño espesor (del orden de $1 \mathrm{~mm}$ ). Además, el proceso de conformación al que se ven sometidos genera unas tensiones residuales de importancia considerable, ya que se produce la plastificación del material para poder ser conformados. Estas dos características los convierten en perfiles con secciones muy esbeltas y susceptibles de presentar problemas de inestabilidad local de abolladura (1). Las normas habituales de cálculo de este tipo de perfiles consideran un cálculo de la resistencia post-crítica mediante la determinación de su sección eficaz. Esta sección eficaz está basada en las teorías de von Karman (20) y permite un análisis postcrítico de su resistencia.
La parte 1-3 del Eurocódigo 3: EN1993-1-3 (21) es de aplicación a este tipo de perfiles conformados en frío de pequeño espesor, estableciendo los valores de las zonas de reducción para la obtención de la sección eficaz. De forma general, para una placa de pequeño espesor sometida a un nivel de tensión constante y condiciones de apoyo en los extremos presentará un ancho reducido dado por la siguiente ecuación (16):

$$
\frac{b_{e f f}}{b}=\sqrt{\frac{\sigma_{c r}}{f_{y}}}\left(1-0,22 \sqrt{\frac{\sigma_{c r}}{f_{y}}}\right)
$$

Donde $b_{e f f}$ es el ancho eficaz de la sección una vez descontada la zona con abolladura, $\sigma_{c r}$ la tensión crítica de pandeo de la placa y $f_{y}$ el límite elástico del material. Esta expresión ha sido obtenida por ajuste experimental y característica de perfiles conformados en frío (1).

Los anchos eficaces de cada zona comprimida se determinarán en función de la ecuación [4], considerando las diferentes condiciones de contorno y distribuciones de carga posibles. El cálculo de la sección eficaz reducida según las indicaciones de EC-3 tiene en cuenta, entre otros, la influencia de las tensiones residuales en este tipo de perfiles conformados en frio

\section{CAMPAÑA EXPERIMENTAL}

\subsection{Geometría de los arcos analizados y materiales}

Las chapas curvas analizadas tienen una sección transversal formada por un perfil trapezoidal, denominado MT-42, de acero conformado en frío y de $0,8 \mathrm{~mm}$ de espesor (Figura 2). El área de la sección transversal es $\mathrm{A}=969 \mathrm{~mm}^{2}$ y el momento de inercia $I_{x}=2,56 \times 10^{-5} \mathrm{~mm}^{4}$.

Las chapas forman un arco circular de radio R, luz L, y longitud de arco S (Figura 3). Los apoyos serán construidos de tal forma que se pueda considerar como un arco biapoyado. Se considerará una carga q uniformemente repartida por unidad de proyección horizontal.

Las chapas han sido fabricadas con acero galvanizado de 250 MPa de límite elástico y 210000 MPa de módulo de elasticidad, siendo la deformación correspondiente al límite elástico $\varepsilon_{\mathrm{y}}=0,0012$.

\subsection{Procedimiento de ensayo e instrumentación}

Se han llevado a cabo ensayos a rotura sobre tres chapas curvas de distintas dimensiones geométricas (Tabla 1). Estos ensayos han consistido en ensayos de carga uniforme mediante sacos de yeso (Figura 4). Para cada una de las distintas geometrías se han ensayado tres muestras, de forma que se ha podido obtener el valor medio de las cargas y su coeficiente de variación.

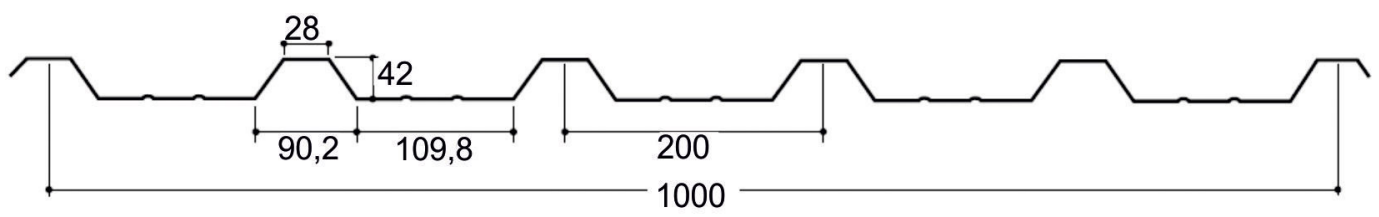

Figura 2. Sección transversal perfil MT-42. 
q

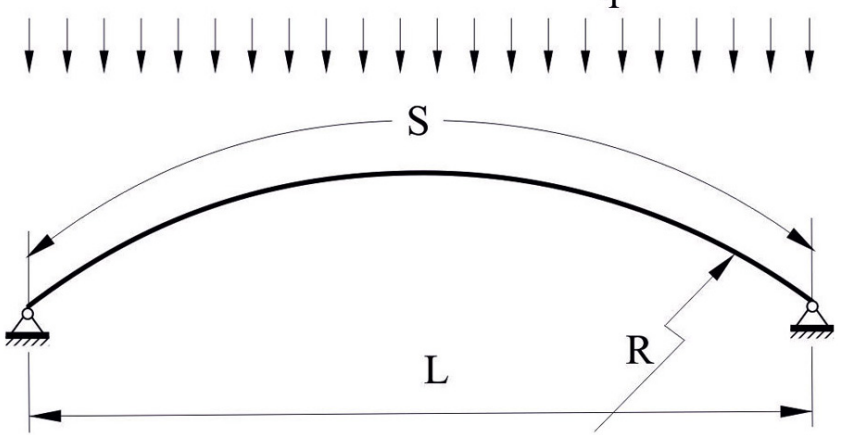

Figura 3. Geometría de las chapas curvas con directriz de arco circular.

Tabla 1. Dimensiones y propiedades geométricas de la chapas ensayadas

\begin{tabular}{|c|c|c|c|c|c|c|}
\hline Chapa & $\begin{array}{l}\mathbf{L u z} \\
\text { (m) }\end{array}$ & $\begin{array}{c}\text { Radio } \\
(\mathbf{m})\end{array}$ & $\begin{array}{c}\text { Espesor } \\
(\mathrm{mm})\end{array}$ & $\begin{array}{l}\text { Inercia } \\
\left(\mathrm{mm}^{4}\right)\end{array}$ & $\begin{array}{c}\text { Área } \\
\left(\mathbf{m m}^{2}\right)\end{array}$ & $\lambda$ \\
\hline 1 & 3,2 & 3,8 & \multirow{3}{*}{0,8} & \multirow{3}{*}{$2,56 \cdot 105$} & \multirow{3}{*}{969} & 43 \\
\hline 2 & 7,0 & 7,0 & & & & 118 \\
\hline 3 & 7,0 & 5,0 & & & & 189 \\
\hline
\end{tabular}

Aunque se dispone de un pórtico de carga para realizar los ensayos, se optó por la utilización del sistema de carga mediante sacos de manera que el arco no tuviera impedido ningún tipo de movimiento en su plano. De esta forma, se puede producir el fallo por pandeo en el plano del arco.

Los sacos han sido pesados y colocados por filas de forma controlada, conociendo en todo momento la carga sobre la chapa. Los sacos se han ido colocando de forma simétrica, de manera que se modifique su modo de fallo lo mínimo posible. Cada saco tiene un peso de $18,8 \mathrm{~kg} \pm 5 \%$. El peso total se ha ido registrando mediante un sistema de adquisición de datos, de forma que cada vez que se coloca un saco se activa un contador que suma su peso. Además se ha recogido la medida del desplazamiento vertical de la clave del arco a través de un transductor tipo LVDT, así como las deformaciones en puntos estratégicos de la chapa a través del pegado de bandas extensométricas en todas las chapas ensayadas.
Las bandas extensométricas se han colocado con el objetivo de medir las deformaciones en la clave del arco y en las proximidades de los apoyos, en la parte superior e inferior de la chapa. Así, se han colocado un total de 10 bandas extensométricas con la distribución indicada en la Figura 5. La chapa presenta unas muescas de doblado en la parte inferior, tal y como se puede apreciar en la Figura 4-izq y. Por este motivo, no se ha podido medir la deformación de la parte inferior de la chapa justo en la clave del arco, colocándose dos bandas simétricas a una distancia de aproximadamente $75 \mathrm{~mm}$ respecto a la clave.

Los apoyos de las chapas se han materializado como apoyos fijos a través de un perfil con sección en forma de omega atornillado a una base metálica con la inclinación adecuada. El desplazamiento transversal de las chapas ha sido impedido a través de la colocación de unas escuadras metálicas de arriostramiento. De esta forma se determina la resistencia en el plano del arco, simulando el efecto de las chapas contiguas (Figura 4-dch).

\subsection{Resultados}

A continuación se representan las curvas carga-desplazamiento y carga-deformación obtenidas durante los ensayos. La representación de los resultados se ha organizado de forma que se muestran los correspondientes a cada una de las distintas chapas ensayadas. Así, en las Figura 6, 7 y 8 se muestran los resultados para las chapas con L-R $=3,2-$ 3,8, 7,0-7,0 y 7,0-5,0 m respectivamente. Como parte de las bandas se han colocado de forma simétrica para el control del comportamiento durante el ensayo, se representan solo los resultados de una de las bandas por cada pareja. Se muestran los resultados de solo una de las muestras de cada chapa.

Como se aprecia en las curvas carga-desplazamiento de las Figura 6, 7 y 8, se observa el efecto de la colocación de los sacos. Inicialmente se empiezan a colocar los sacos en los extremos, por lo que se produce un levantamiento de la clave del arco que se manifiesta en la curva carga-desplazamiento obtenida. En todos los ensayos se ha completado al menos una capa de sacos de forma que cubra toda la superficie de la chapa.
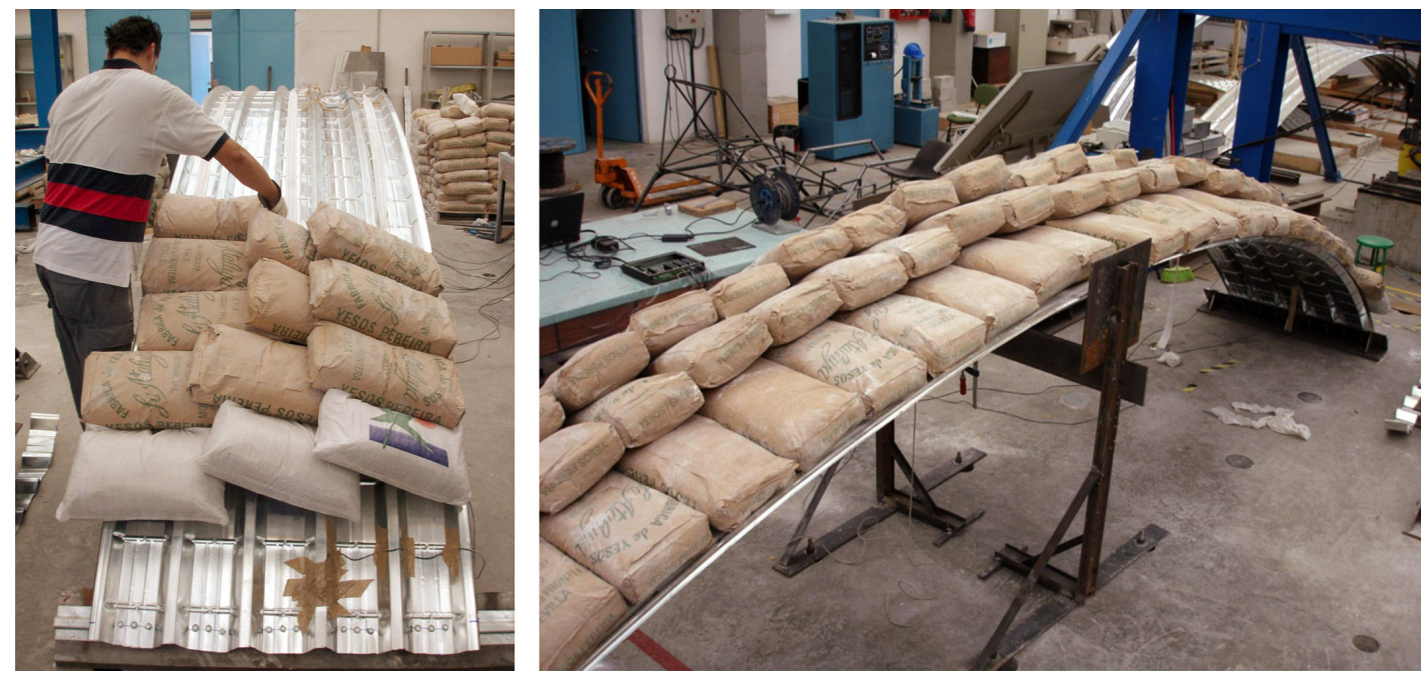

Figura 4. Ensayos de carga uniforme. 

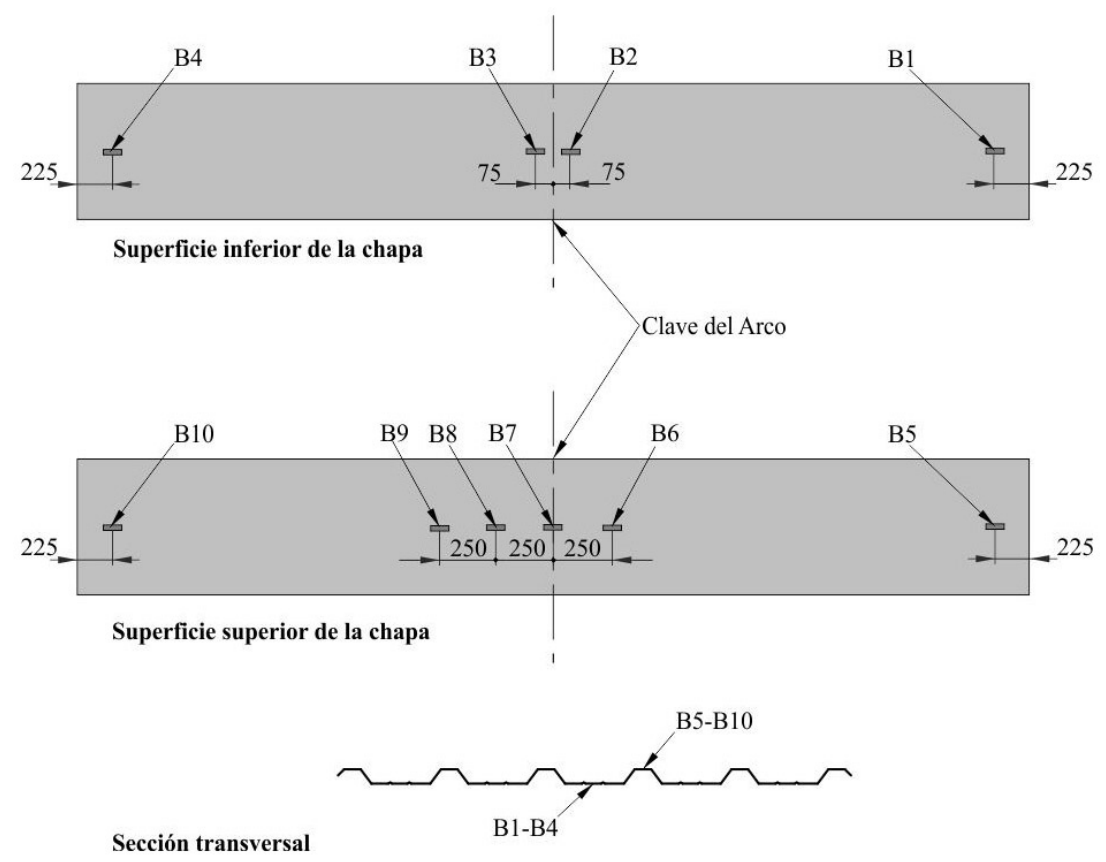

Figura 5. Distribución de las bandas colocadas.
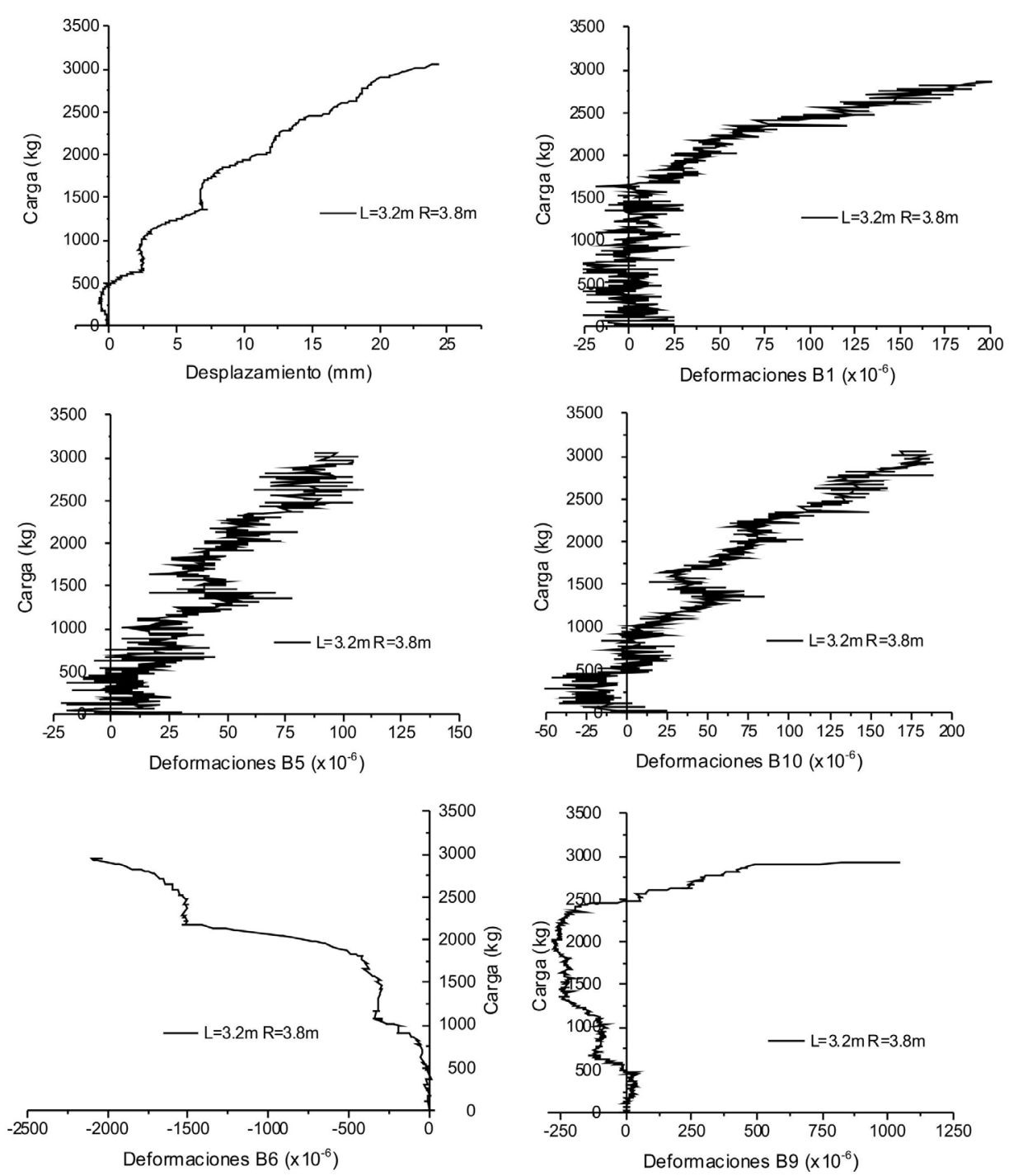

Figura 6. Curvas carga-desplazamiento y carga-deformaciones para la chapa de $\mathrm{L}=3,2 \mathrm{~m}$ y $\mathrm{R}=3,8 \mathrm{~m}$. 

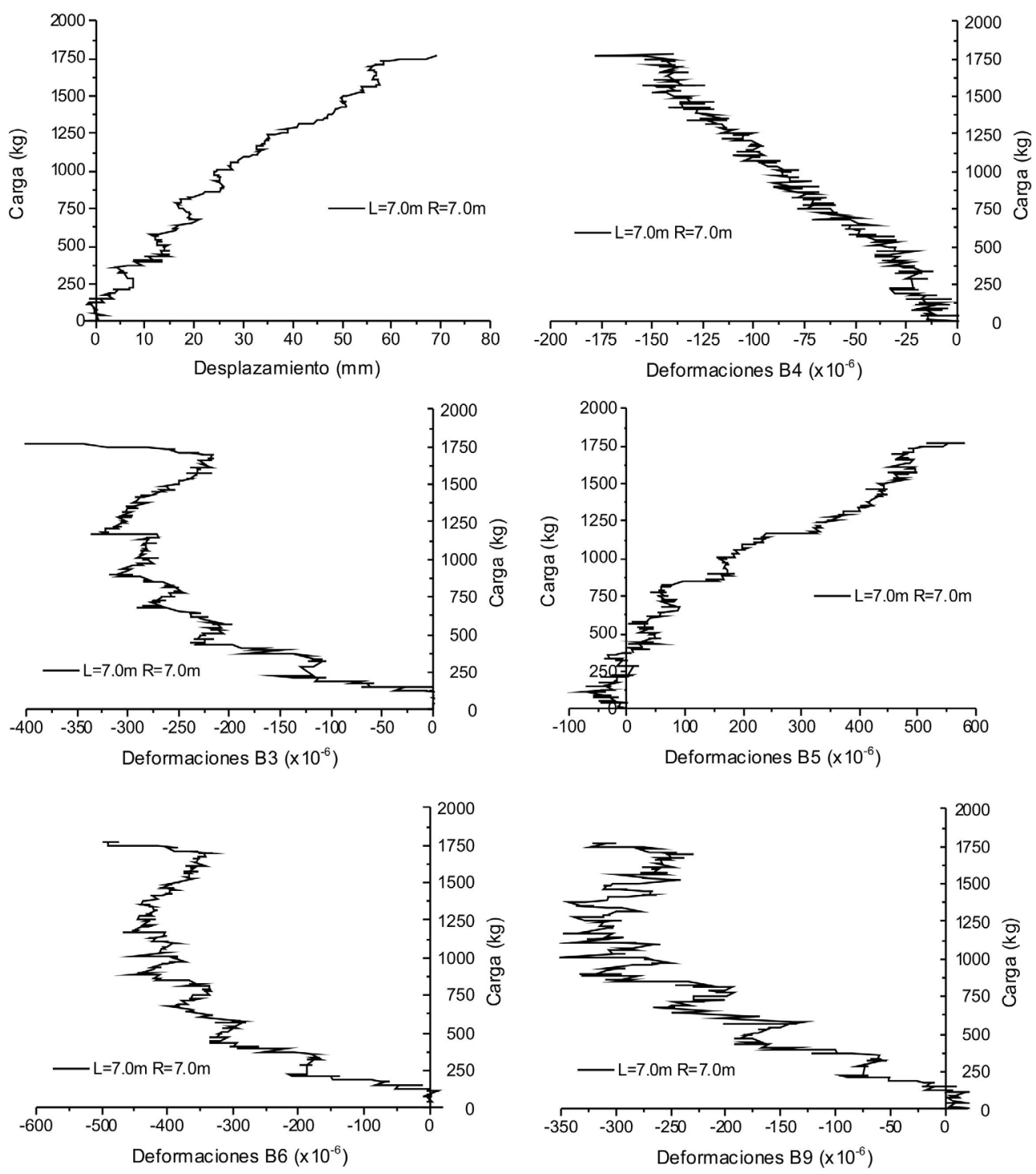

Figura 7. Curvas carga-desplazamiento y carga-deformaciones para la chapa de $\mathrm{L}=7,0 \mathrm{~m}$ y $\mathrm{R}=7,0 \mathrm{~m}$.

Las deformaciones medidas por las bandas extensométricas son de tracción (positivas) o compresión (negativas), dependiendo de la posición de la banda (superior o inferior) y de los esfuerzos originados en la zona donde están colocadas. En aquellas bandas que estando colocadas en la misma zona del arco, pero una perteneciente a la fibra superior de la sección y otra a la inferior, tengas ambas deformaciones de compresión corresponde a una zona con un estado de cargas donde predomina la compresión respecto a la flexión.

En la Tabla 2 se muestran las cargas últimas medias experimentales, $q_{\text {exp. }}$, obtenidas para cada chapa, con indicación del coeficiente de variación, y el menor valor obtenido de carga, $q_{\text {exp.min }}$. Asimismo, se muestra la indicación de si se ha llegado a medir una deformación superior al límite elástico, antes del colapso, en alguna de las bandas extensométricas colocadas y la flecha máxima.

Los modos de fallo observados han sido generalmente de inestabilidad global por pandeo claramente asimétrico para el caso de las chapas 2 y 3 con mayor esbeltez y pandeo simétrico para el caso 1 (arco de menor esbeltez). Este efecto se ha constatado adicionalmente mediante los valores de las deformaciones medidas por las bandas extensométricas instantes previos al colapso. Como se observa en la Tabla 2, en ningún caso se ha alcanzado el límite elástico en el acero antes de producirse el fallo de la chapa.

Tabla 2. Cargas últimas experimentales.

\begin{tabular}{|c|c|c|c|c|c|}
\hline Chapa & $\begin{array}{c}\mathbf{q}_{\text {exp.m }} \\
(\mathbf{d a N} / \mathbf{m})\end{array}$ & $\begin{array}{c}\mathbf{q}_{\text {exp.min }} \\
(\mathbf{d a N} / \mathbf{m})\end{array}$ & $\begin{array}{c}\text { Límite } \\
\text { elástico }\end{array}$ & $\begin{array}{c}\text { Flecha } \\
\text { (mm) }\end{array}$ & $\begin{array}{c}\text { Modo } \\
\text { de fallo }\end{array}$ \\
\hline 1 & $9,70 \pm 6 \%$ & 9,11 & NO & 24,4 & Pandeo simétrico \\
\hline 2 & $2,93 \pm 9 \%$ & 2,67 & NO & 90,2 & Pandeo asimétrico \\
\hline 3 & $1,76 \pm 15 \%$ & 1,50 & NO & 89,5 & Pandeo asimétrico \\
\hline
\end{tabular}



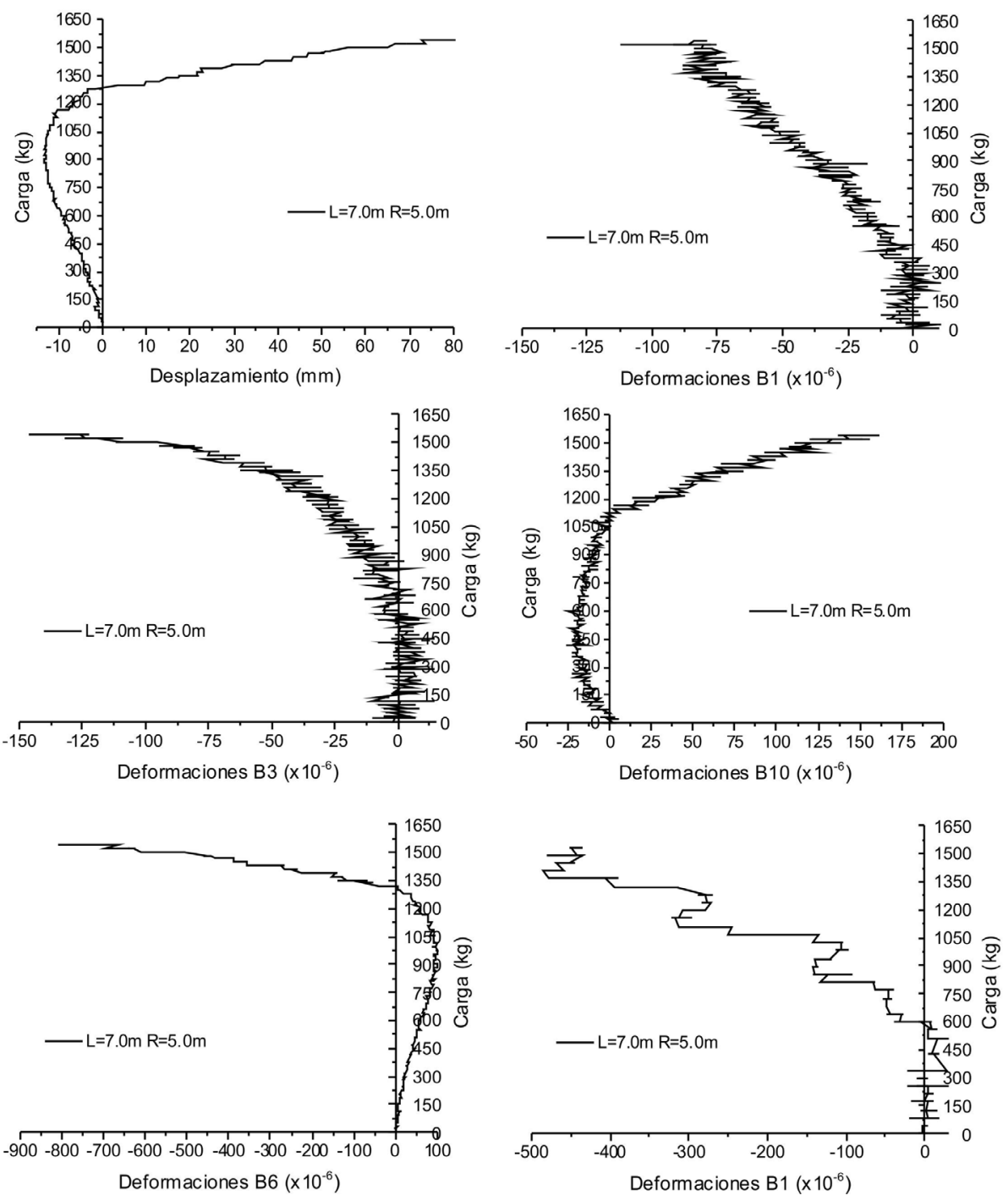

Figura 8. Curvas carga-desplazamiento y carga-deformaciones para la chapa de L = 7,o m y R= 5, $\mathrm{O}$.

\section{ESTUDIO NUMÉRICO}

\subsection{Descripción del modelo numérico}

Se ha llevado a cabo un estudio numérico mediante un modelo de elementos finitos realizado con el programa RFEM de la empresa DLUBAL (22). La discretización de las chapas curvas se ha realizado mediante elementos lámina tipo MITC4, con la geometría correspondiente a cada una de las chapas ensayadas experimentalmente. En la Figura 9-izq. se muestra la discretización de la malla realizada para el caso de la chapa 1.

Se ha considerado un material correspondiente al acero, con un límite elástico de $210000 \mathrm{MPa}$, un coeficiente de Poisson de 0,3 y comportamiento elástico y lineal exclusivamente. Las condiciones de contorno y las cargas son las correspondientes a los ensayos experimentales, apoyos fijos en los extremos y carga uniformemente distribuida.

\subsection{Resultados}

Se ha realizado un estudio de estabilidad de cada arco, considerando efectos de segundo orden. Este cálculo considerando no linealidades geométricas responde a las indicaciones de Pi y Bradford (10) (12) respecto a la conveniencia de consideración de los efectos de segundo orden en el caso de arcos circulares. Los primeros modos de pandeo obtenidos corresponden a casos de pandeo asimétrico para las chapas más esbeltas, lo que es consistente con lo establecido por Bradford et al. (12) para arcos de elevada esbeltez. En la Figura 9-dcha. se muestra el modo de pandeo obtenido mediante elementos finitos para la chapa 2.

En la Tabla 3 se muestran los resultados correspondientes a las cargas críticas obtenidas para cada una de los arcos analizados, así como la esbeltez del arco según la ecuación [1]. Estas cargas representan el valor de la carga máxima obtenida en los modelos numéricos, para un cálculo con no-linealidad geométrica y comportamiento elástico-lineal del material y que corresponden con las cargas numéricas de pandeo global de los arcos analizados.

Como se observa en los valores obtenidos en la Tabla 3, a medida que aumenta la esbeltez del elemento disminuye el valor de la carga que produce el fallo por pandeo global. Para el caso de la chapa de menor esbeltez se observa que la 

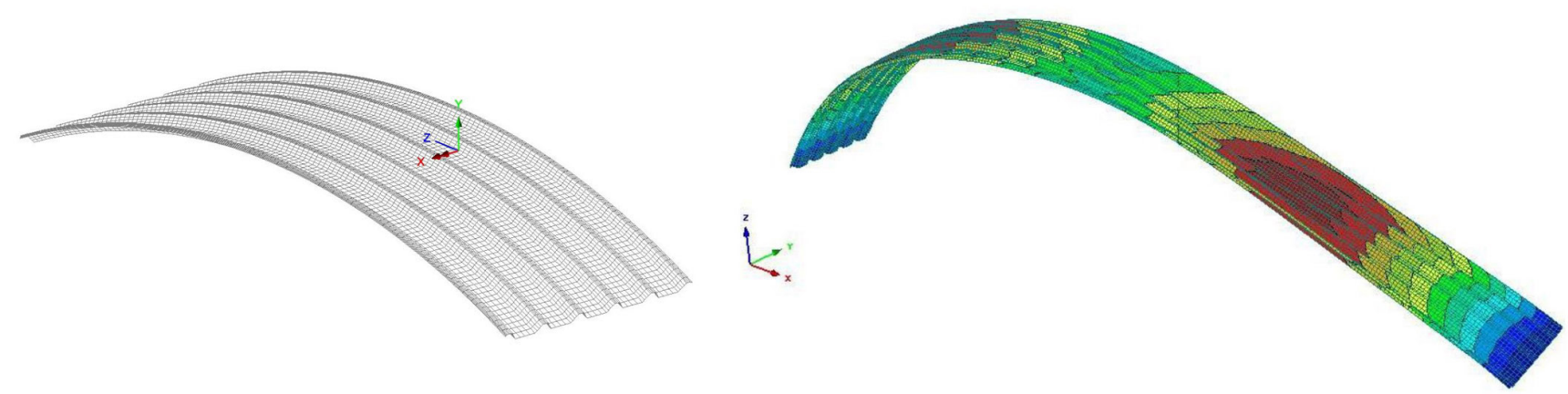

Figura 9. Discretización empleada en la modelización de la chapa 1 (izq.) y pandeo asimétrico obtenido para la chapa 2 (dcha.)

Tabla 3. Valores de la carga crítica obtenidas con MEF.

\begin{tabular}{|c|c|}
\hline Chapa & $\boldsymbol{q}_{\mathbf{c r}}(\mathbf{k N} / \mathbf{m})$ \\
\hline 1 & 46,90 \\
\hline 2 & 2,89 \\
\hline 3 & 2,59 \\
\hline
\end{tabular}

carga crítica es muy elevada, lo que está en consonancia con el modo de fallo observado experimentalmente (es la única chapa que presentó fallo por pandeo simétrico). Por tanto, los modelos numéricos permiten constatar los resultados experimentales obtenidos y el modo de fallo observado durante la realización de los ensayos en laboratorio.

\section{PROCEDIMIENTO PARA LA OBTENCIÓN DE CARGAS ÚLTIMAS}

La metodología de cálculo propuesta comparte su filosofía con los procedimientos recogidos en el EC3 para el cálculo de elementos rectos sometidos a compresión. De esta forma, en primer lugar ha de obtenerse la carga última teórica por criterio de agotamiento resistente. Posteriormente, esta carga se reducirá en función de un coeficiente reductor, relacionado con los resultados experimentales y que contempla fenómenos de inestabilidad global, obteniéndose así la carga real última de la chapa.

\subsection{Determinación de esfuerzos en arcos de circunferencia}

Para la obtención de la carga última teórica por resistencia de la chapa es necesaria la determinación analítica de los esfuerzos sobre el arco. En el caso de un arco simétrico biapoyado, de ángulo de apertura $\alpha$, sometido a una carga uniforme por proyección de superficie horizontal (Figura 10), se tiene que el esfuerzo axil, $N$, cortante, $Q$, y flector, $M$, para una sección con un ángulo $\theta$ con respecto a la vertical, vienen dados por:

$$
\begin{gathered}
N=F_{x} \cdot \cos (\theta)-F_{y} \cdot \operatorname{sen}(\theta)+q \cdot R \cdot(\alpha-\theta) \cdot \operatorname{sen}(\theta) \\
Q=F_{y} \cdot \operatorname{sen}(\theta)-F_{x} \cdot \cos (\theta)-q \cdot R \cdot(\alpha-\theta) \cdot \cos (\theta) \\
M=F_{y} \cdot R \cdot[\operatorname{sen}(\alpha)-\operatorname{sen}(\theta)]+F_{x} \cdot R \cdot[\cos (\theta)-\cos (\alpha)]- \\
-q \cdot R^{2} \cdot \int_{\theta}^{\alpha}[\operatorname{sen}(\alpha)-\operatorname{sen}(\theta)] \cdot d \beta
\end{gathered}
$$

siendo:

$F_{x}, F_{y}$ reacciones horizontal y vertical en los apoyos, cuyas expresiones se encuentran en diversos manuales (23).

\subsection{Determinación de las cargas últimas frente a ELU de resistencia}

La determinación de la carga máxima se ha realizado mediante un análisis elástico sobre la sección eficaz, ya que la sección transversal es de clase 4 (2). En la Tabla 4 se muestran los resultados obtenidos de carga máxima uniforme, $q_{u . e f}$ así como su relación con la carga de fallo por pandeo global.

Como era de esperar, las cargas últimas analíticas son superiores a las cargas últimas experimentales.

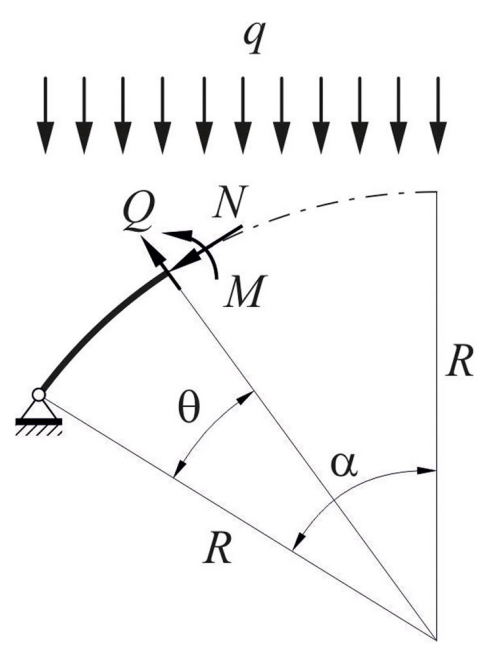

\begin{tabular}{|c|c|c|c|c|c|}
\hline Chapa & $\begin{array}{c}\begin{array}{c}\text { Área } \\
\text { efectiva } \\
\left(\mathbf{m m}^{2}\right)\end{array}\end{array}$ & $\begin{array}{c}\text { Inercia } \\
\text { efectiva } \\
\left(\mathrm{mm}^{4}\right)\end{array}$ & $\underset{(\mathbf{k g} / \mathbf{m})}{\boldsymbol{q}_{u, f}}$ & $\boldsymbol{q}_{u . e f} / \boldsymbol{q}_{\text {exp }}$ & $\boldsymbol{q}_{u . e f} / \mathbf{q}_{\text {cr }}$ \\
\hline 1 & 522 & $1,74 \cdot 105$ & 3195 & 3,51 & 0,68 \\
\hline 2 & 591 & $1,75 \cdot 105$ & 1166 & 4,37 & 3,61 \\
\hline 3 & 569 & $1,74 \cdot 105$ & 1435 & 9,57 & 4,65 \\
\hline
\end{tabular}

Figura 10. Esfuerzos en un arco circular sometido a carga uniformemente distribuida por unidad de superficie proyectada.

Tabla 4. Cargas últimas obtenidas por criterio de resistencia. 


\section{5•3. Obtención de un coeficiente reductor}

En la Tabla 4 se muestra la relación existente entre la carga última por resistencia y la carga última obtenida de forma experimental. La inversa de esta relación sería el coeficiente reductor por el que debe multiplicarse la carga teórica para obtener la carga última real considerando fenómenos de inestabilidad. Teniendo en cuenta la geometría de los arcos ensayados puede llegar a obtenerse una expresión que relaciona los valores de este coeficiente reductor en función de la luz y el radio de las chapas ensayadas. La ecuación [8] muestra el coeficiente de reducción, aplicando un coeficiente de minoración $\gamma_{\text {M.e }}=1,16$ a los resultados experimentales.

$$
X=0,108+(R-5) \cdot 0,0295+(7-L) \cdot 0,0454
$$

En esta expresión $\mathrm{R}$ es el radio de curvatura de la chapa y $\mathrm{L}$ la distancia entre apoyos de la misma. Ambas magnitudes deben introducirse en metros.

Respecto al coeficiente de minoración adoptado, el número de muestras ensayadas no es suficiente para la realización de un estudio estadístico para su determinación, por lo que se ha determinado un valor obtenido de forma simplificada, similar a lo indicado en (24). Así, se ha considerado que la resistencia característica de las chapas se obtiene multiplicando por 0,9 el menor valor de la carga última experimental, que aplicando posteriormente un coeficiente de minoración del material correspondiente a Estado Límite de Inestabilidad de $\gamma_{\text {M.2 }}=1,05$ se obtiene que $\gamma_{\text {M.e }}=1,05 / 0,9=1,16$.

Este coeficiente reductor empírico tiene en cuenta los fenómenos de inestabilidad en función de las cargas experimentales. Esta filosofía es similar a la propuesta en los Eurocódigos a través de las curvas europeas de pandeo. Con la intención de obtener un coeficiente reductor en función únicamente de la esbeltez del elemento, se representa la ecuación [8] en función de dicha esbeltez. Como la esbeltez dada por la ecuación [1] depende de la luz y del radio simultáneamente, se ha representado el coeficiente de reducción en función de la esbeltez de forma paramétrica para distintos valores de la luz del arco circular (Figura 11).

Como se observa en la Figura 11, se ha representado el factor de reducción de la carga última por resistencia del arco

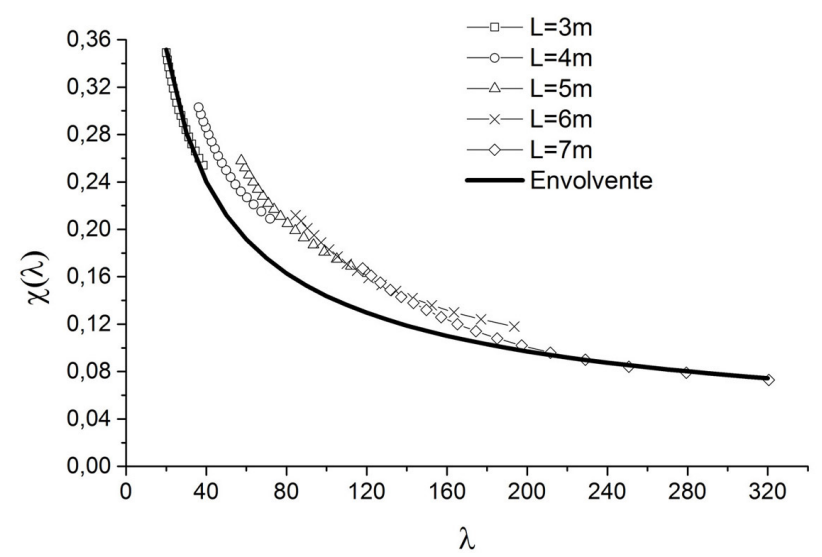

Figura 11. Envolvente del factor de reducción en función de la esbeltez del arco. en función de la esbeltez del mismo mediante la ecuación [8] para luces de 3, 4, 5, 6 y $7 \mathrm{~m}$ y radios comprendidos entre 3,8 y $7 \mathrm{~m}$. Se aprecia que existen valores distintos del radio y la luz del arco para los que coincide la esbeltez y que proporcionan diferentes valores del factor de reducción. Con el objetivo de unificar el valor del factor de reducción en función de la esbeltez del arco se traza una envolvente inferior a las distintas familias de curvas obtenidas para cada luz. La forma de esta envolvente es muy parecida a la que presentan las curvas europeas de pandeo, siendo la expresión que mejor se ajusta la siguiente:

$$
\chi(\lambda)=\frac{1.994}{(1+\lambda)^{0.569}}
$$

Esta envolvente proporciona valores del factor de reducción que son únicos para cada valor de la esbeltez del arco y que se encuentran del lado de la seguridad para cualquier caso.

Una vez determinada la función empírica definida por la ecuación [9], el procedimiento de obtención de las cargas últimas para cualquier valor del radio y la luz del arco circular es de fácil aplicación. El procedimiento de cálculo consistirá en obtener el valor de la carga externa $q_{\text {u.ef }}$ que produce el agotamiento elástico de la sección eficaz y posteriormente aplicar el factor de reducción que tiene en cuenta el fenómeno de inestabilidad por pandeo global del arco en función de la esbeltez del mismo, de forma que:

$$
q_{u . b}=q_{u . e f} \chi(\lambda)
$$

Los límites de aplicación de la metodología propuesta se establecen en función de los valores límite de las luces y radios de las chapas ensayadas. De acuerdo a los ensayos efectuados, las luces límite serían $3 \mathrm{~m}$ y $7 \mathrm{~m}$ y los radios deberían estar comprendidos entre $3,8 \mathrm{~m}$ y $7 \mathrm{~m}$. No obstante, tal y como se observa en la Figura 11 la función del factor de reducción obtenida como envolvente inferior es monótona decreciente, por lo que podría establecerse una limitación respecto a la esbeltez de los arcos. Los valores límite de la esbeltez para el caso analizado serían una esbeltez mínima de $\lambda_{\min }=20$ y una esbeltez máxima de $\lambda_{\max }=320$. Debe remarcarse que los resultados obtenidos son válidos únicamente para chapas con una sección transversal como la indicada en la Figura 2 y con un espesor de $0,8 \mathrm{~mm}$, siendo posible realizar una extrapolación conservadora a chapas de mayor espesor. La consideración de chapas con distinta sección transversal modificaría los valores de las esbelteces b/t, lo que podría afectar negativamente al pandeo local de la misma, interactuando con el pandeo global. Por otro lado, el ensayo de un mayor número de chapas distintas aumentaría la fiabilidad del procedimiento aquí propuesto, siendo el número de casos analizados en este trabajo de 3 y considerado como el mínimo número de casos a analizar para el desarrollo del procedimiento.

\section{DETERMINACIÓN DEL RADIO DE CURVATURA MÁS ADECUADO}

En la Figura 12 se muestran los valores de las cargas últimas, obtenidas según el procedimiento propuesto, en función de la luz para distintos valores del radio de curvatura. Así, se han analizado configuraciones de arcos circulares con luces comprendidas entre 3,0 y 7,0 m y radios de curvatura del arco entre 3,5 y 9,o m. 


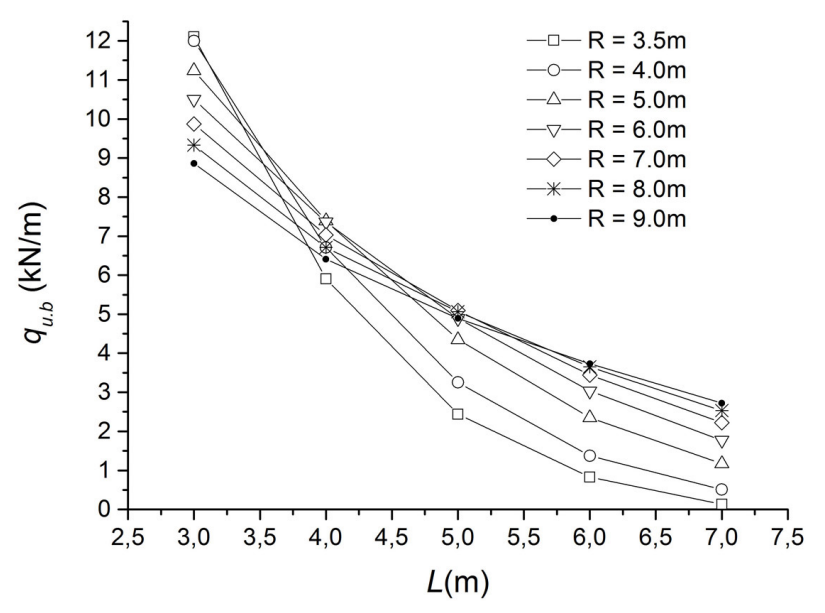

Figura 12. Cargas últimas de las chapas curvas para distintas geometría de arcos circulares.

Tabla 5. Valores más adecuados del radio de curvatura para distintas luces entre apoyos.

\begin{tabular}{|c|c|c|}
\hline $\begin{array}{c}\text { Luz entre apoyos } \\
\text { de la chapa (m) }\end{array}$ & $\begin{array}{c}\text { Radio óptimo de } \\
\text { curvatura (m) }\end{array}$ & $\begin{array}{c}\text { Esbeltez } \\
\text { del arco }\end{array}$ \\
\hline 3,0 & 3,5 & 41,7 \\
\hline 4,0 & 5,0 & 51,5 \\
\hline 5,0 & 7,0 & 56,7 \\
\hline 6,0 & 9,0 & 63,1 \\
\hline 7,0 & 9,0 & 87,9 \\
\hline
\end{tabular}

Como se desprende de las curvas mostradas en la Figura 12 existen valores de los radios de curvatura de las chapas que presentan los mayores valores de las cargas últimas en función de la luz que se esté considerando. En la Tabla 5 se muestran los valores más adecuados de los radios de curvatura en función de la distancia entre apoyos del arco circular.

Como se observa en la Tabla 5, a medida que aumenta la luz del arco es necesario aumentar en mayor proporción el radio óptimo de curvatura. Además, también se observa que la esbeltez más adecuada del arco aumenta con la luz entre apoyos, de forma que para una luz de 7,0 m la esbeltez óptima del arco llega a duplicar la correspondiente a 3,0 m. Este efecto es contrario a lo obtenido para el coeficiente reductor de pandeo, que disminuye cuando aumenta la esbeltez del arco. La explicación a este fenómeno se debe a que al aumentar la distancia entre apoyos también lo hacen los esfuerzos de flexión sobre el arco y por tanto, disminuye notablemente la carga última por resistencia. Por otro lado, un aumento del radio disminuye estos importantes esfuerzos de flexión y aunque también aumenta la esbeltez, su efecto es más notable.

\section{CONCLUSIONES}

A la vista de los resultados obtenidos se pueden establecer las siguientes conclusiones:

Se han obtenido las cargas últimas experimentales de tres arcos circulares con distinta geometría. El arco de menor esbeltez ha experimentado fallo por pandeo simétrico del mismo. En el resto de arcos ensayados, con una esbeltez muy superior, el modo de pandeo ha sido asimétrico. Estas conclusiones son consistentes con lo establecido por Pi y Bradford.

Se ha determinado una función del factor de reducción de la carga última por resistencia sobre la sección eficaz considerando inestabilidad por pandeo en función de la esbeltez del arco. La forma de dicha función es similar a la que presentan las curvas europeas de pandeo recogidas en los Eurocódigos. Los fenómenos de inestabilidad local se consideran mediante la sección reducida.

En función de los resultados experimentales se ha establecido una metodología de cálculo para la estimación de la carga última en arcos con diferentes valores de la luz y el radio.

El radio de curvatura de los arcos que proporciona las mayores resistencias aumenta más que proporcionalmente con la distancia entre apoyos, debido a que un aumento del radio disminuye notablemente los esfuerzos de flexión producidos por el aumento de la luz entre apoyos.

\section{AGRADECIMIENTOS}

Los autores desean agradecer a la empresa HIANSA, S.A. la financiación para la realización del presente trabajo, a través del proyecto: Estudio Numérico y Experimental de Chapas Curvas (ES-0463/2006). Asimismo, los autores agradecen a D. Emilio Javier Gómez Álvarez su inestimable colaboración en la realización de los trabajos experimentales.

\section{REFERENCIAS}

(1) Yu, W.W., LaBoube, R.A. (2010). Cold-formed steel design, $4^{\text {a }}$ Edición. EEUU: John Wiley \& Sons. Doi: http://dx.doi. org/10.1002/9780470949825.

(2) AENOR. (2004). UNE-EN 1993-1-1 Eurocódigo 3: Proyecto de Estructuras de Acero. Parte 1-1: Reglas generales y reglas para edificación. Asociación Española de Normalización (AENOR).

(3) Dimopoulos, C.A., Gantes, C.J. (2008). Design of circular steel arches with hollow circular cross-sections according to EC3. Journal of Constructional Steel Research, 64(10): 1077-1085, doi: http://dx.doi.org/10.1016/j.jcsr.2007.09.009.

(4) Fung, Y.C., Kaplan, A. (1952). Buckling of low arches of curved beams of small curvature. TN, 2840. Washington: National Advisory Committee for Aeronautics.

(5) Timoshenko, S.P., Gere, J.M. (1961). Theory of elastic stability. New York: Mc Graw Hill.

(6) Gjelsvik, A., Bodner, S.R. (1962). Energy criterion and snap-through buckling of arches. Journal of Engineering Mechanics ASCE, 88(EM5): 87-134.

(7) Pi, Y-L., Bradford, M.A. (2013). Nonlinear elastic analysis and buckling of pinned-fixed arches. International Journal of Mechanical Sciences, 68: 212-223, doi: http://dx.doi.org/10.1016/j.ijmecsci.2013.01.018. 
(8) Attard, M.M.; Zhu, J., Kellermann, D. (2013). In-plane buckling of circular arches and rings with shear deformations. Archive of Applied Mechanics, 83(8): 1145-1169: doi: http://dx.doi.org/10.1007/s00419-013-0740-y.

(9) Pi, Y-L., Changyong, L., Bradford, M.A., Zhang, S. (2012). In-plane strength of concrete-filled steel tubular circular arches. Journal of Constructional Steel Research, 69(1): 77-94, doi: http://dx.doi.org/10.1016/j.jcsr.2011.08.008.

(10) Pi, Y-L., Bradford, M.A., Uy, B. (2002). In-plane stability of arches. International Journal of Solids and Structures, 39(1): 105-125, doi: http://dx.doi.org/10.1016/Soo20-7683(01)o0209-8.

(11) Pi, Y-L., Bradford, M.A., Tin-Loi, F. (2007). Nonlinear analysis and buckling of elastically supported circular shallow arches. International Journal of Solids and Structures, 44(7-8): 2401-2425, doi: http://dx.doi.org/10.1016/j.ijsolstr.2006.07.011.

(12) Bradford, M.A., Uy, B., Pi, L-Y. (2002). In-Plane elastic stability of arches under a central concentrated load. Journal of Engineering Mechanics ASCE, 128(7): 710-719, doi: http://dx.doi.org/10.1061/(ASCE)0733-9399(2002)128:7(710).

(13) Pi, Y-L., Trahair, N.S. (1996). In-plane ineslatic buckling and strengths of steel arches. Journal of Structural Engineering ASCE, 122(7): 734-747, doi: http://dx.doi.org/10.1061/(ASCE)0733-9445(1996)122:7(734).

(14) Pi, Y-L., Trahair, N.S. (2000). Inelastic lateral buckling strength and design of steel arches. Engineering Structures, 22(8): 993-1005, doi: http://dx.doi.org/10.1016/So141-0296(99)00032-2.

(15) Hodges, H.H. (1999). Non-linear inplane deformation and buckling of rings and high arches. International Journal of Non-Linear Mechanics, 34(4): 723-737, doi: http://dx.doi.org/10.1016/Soo20-7462(98)ooo50-X.

(16) Trahair N.S., Bradford M.A., Nethercot D.A., Gardner L. (2008). The behavior and design of steel structures to EC3. EEUU y Canada: Taylor and Francis.

(17) Kuranishi, S., Yabuki, T. (1979). Some numerical estimation of the ultimate in plane strength of two-hinged steel arches. En Proceedings of the Japan Society of Civil Engineers, 287: 155-158, doi: http://dx.doi.org/10.2208/ jscej1969.1979.287_155.

(18) Verstappen, I., Snijger, H., Bijlaard, F.S.K., Steenbergen, H.M.G.M. (2012). Design rules for steel arches-in-plane stability. Journal of Constructional Steel Research, 46(1-3): 125-126, doi: http://dx.doi.org/10.1016/So143-974X(98)oo022-4.

(19) Dimopoulos, C.A., Gantes, C.J. (2008). Nonlinear in-plane behavior of circular steel arches with hollow circular cross-section. Journal of Constructional Steel Research, 64(12): 1436-1445, doi: http://dx.doi.org/10.1016/j.jcsr.2008.01.005.

(20) Argüelles-Alvarez, R., Argüelles-Bustillo, R., Arriaga F., Atienza, J.R. (1999). Estructuras de Acero. 1: Cálculo, Norma Básica y Eurocódigo. Madrid: Bellisco.

(21) AENOR. (2012). UNE-EN 1993-1-3 Eurocódigo 3: Proyecto de Estructuras de Acero. Parte 1-3: Reglas generales y reglas adicionales para perfiles y chapas de paredes delgadas conformadas en frío. Asociación Española de Normalización (AENOR).

(22) Dlubal. (2013). RFEM5 Manual-Program Description. Dlubal Software GmbH. https://www.dlubal.com/en/rfem-5xx. aspx.

(23) Young, W. y Budynas, R. (2011). Roark's formulas for stress \& strain. 8a Edición. Singapur: Mc Graw Hill.

(24) AENOR. (2013). UNE-EN199-1-1 Eurocódigo 4: Proyecto de Estructuras Mixtas de Acero y Hormigón. Parte 1-1: Reglas generales y reglas para edificación. Asociación Española de Normalización (AENOR). 